\title{
De la dynamique des systèmes au pilotage opérationnel de l'imprévisible : l'apport de la cybernétique
}

\author{
From dynamics of systems to operational management of the \\ unpredictable: the contribution of cybernetics
}

\author{
Yvonne Auberlet de Chelle ${ }^{1}$ \\ ${ }^{1}$ CADEV, France, yauberlet@meteorix.fr
}

\begin{abstract}
RÉSUMÉ. Le déroulement des opérations dans une entreprise est de plus en plus perturbé par des éléments imprévisibles de leur environnement interne ou externe. Une réponse à cette situation peut être fondée sur la dynamique des systèmes. Celle-ci, initialement conçue pour fournir une méthode d'analyse de processus de gestion, a prospéré surtout dans des domaines autres que celui du management industriel : analyse de systèmes urbains, modèles du monde, modèle de l'économie américaine... L'article montre que, en marge de cet essor académique, le retour à l'esprit initial inspiré par la cybernétique a permis de stimuler la modélisation d'une chaîne d'activité industrielle, sur la base de la description et de l'exploration des propriétés dynamiques de ses modèles. Grâce à la représentation réaliste de ces derniers en termes de dynamiques temporelles de flux irriguant un environnement industriel considéré comme un système non linéaire dynamique réagissant à des perturbations paramétrées à caractère aléatoire, la simulation décrite constitue un puissant moyen d'aide à la décision, tant opérationnelle (au sens du court terme) que stratégique (au sens du long terme).
\end{abstract}

ABSTRACT. The course of operations in a company is increasingly disrupted by unpredictable elements experienced in their internal or external environment. A response to this situation may be based on the dynamics of systems. The latter, initially conceived to provide a method of analysis of management processes, has flourished especially in fields other than industrial management: analysis of urban systems, world models, American economy models... This paper shows that, on the sidelines of this academic boom, the reactivation of the initial spirit inspired by cybernetics has helped to stimulate the modeling of a chain of industrial activity, based on the description and exploration of the dynamic properties of its models. Thanks to the realistic representation of the latter in terms of temporal dynamic flows irrigating an industrial environment considered as a dynamic non-linear system reacting to randomized parameterized perturbations, the simulation described is a powerful means of decisional support, both operational (in the short-term view) and strategic (in the long-term view).

MOTS-CLÉS. Systèmes Dynamiques, Flux économiques, Modélisation, Cybernétique, Pilotage, Imprévisible, Cygnes Noirs, Stratégie.

KEYWORDS. Dynamic Systems, Economic flows, Modeling, Cybernetic, Steering, Unpredictable, Black Swans, Strategy.

\section{Introduction}

Les Organisations économiques, quelle que soit la nature de leurs activités, sont liées à leur environnement avec lequel elles forment un "système » vivant et interactif. Ainsi, une part d'imprévisible s'impose à elles sous forme d'innovations inattendues, de nouveaux entrants, mais aussi d'événements sociaux, météorologiques, épidémiques perturbateurs. Par ailleurs, le fonctionnement économique n'est pas uniforme et il subit des aléas, des irrégularités de fonctionnement, des erreurs, des pannes, des conflits. Ces imprévus modifient le fonctionnement attendu de l'organisation et ils peuvent provoquer des déséquilibres.

Tous ces imprévus constituent une réalité incontournable et il importe de mettre en place les moyens de les manager pour les maitriser, en apprenant à anticiper, à créer des coopérations entre personnes et services, à aménager les modes de travail. Pour cela, il s'agit d'évaluer le coût du risque à partir des faits connus et de chiffrer ses conséquences opérationnelles. Encore faut-il disposer d'un outil 
d'évaluation et de comparaison pour décider ce que l'on voudra faire en vue d'optimiser les résultats, plutôt que de faire des expériences directes et risquées. Une plateforme de simulation systémique des flux économiques et le leurs interactions est indispensable pour obtenir des bases de décision.

Il nous faut aussi penser aux changements profonds, subits, inattendus et durables d'un futur désormais très proche : changements économiques, sociologiques et technologiques, modifications des produits, des services à fournir, des marchés, d'environnement social et régional. L'existant devient obsolète et les impacts sociologiques des changements sont profonds.

Un des enjeux de l'économie actuelle est de créer les conditions de réussite des transformations inéluctables qui nous attendent, avec une vision commune du futur et la maîtrise des interactions entre les savoir-faire et les produits, les ressources techniques et humaines, les activités et les finances, jalonnées dans le temps, avec des indicateurs de déséquilibres possibles. Il faut imaginer les scénarios de futurs possibles, anticiper de nouvelles organisations et nous préparer aux impacts organisationnels et sociaux. Et prévoir les risques de perturbations de ces prévisions, mettre en place des mécanismes d'alarme.

A l'intérieur, les organisations se heurtent à des luttes de pouvoir, des contradictions d'intérêts, des visions différentes, un manque de maîtrise des interactions entre les savoir-faire, les activités, les ressources et les finances. Pourtant tout le monde a avantage à ce que, in fine, les résultats soient positifs. Des dispositifs de concertation doivent être mis en place pour préparer le futur et établir des plans, puis assurer, ensemble, leur bon déroulement. Les plans décidés doivent être assortis de mécanismes de contrôle et d'alarmes sur les déviations possibles : on ne sait quand celles-ci surviendront, mais la probabilité de leur émergence devient d'autant plus forte que nous vivons un changement profond de l'économie et des technologies. Il faut ainsi imaginer les risques de modifications inattendues des environnements techniques, sociaux, économiques, et leurs impacts possibles sur le fonctionnement. La maîtrise et l'équilibrage des organisations économiques dans le temps passent par l'intégration de ce référentiel des déséquilibres imaginés. La modélisation du fonctionnement futur et l'organisation de son équilibrage inclut les dispositifs nécessaires à la maitrise de ces déséquilibres possibles. Une plateforme de simulation systémique de scénarios du futur permet d'évaluer les résultats de chaque scénario et obtenir des bases de décision.

Le présent article va s'articuler autour d'un adossement à la dynamique des systèmes : seront d'abord convoquées les bases théoriques des systèmes dynamiques. Ensuite, nous montrerons que, parallèlement au développement très large en termes de domaines des sciences de la matière et de la vie, à visée principalement d'expansion des connaissances, l'approche initiale a donné lieu à la formalisation d'une démarche appliquée en pratique au management organisationnel industriel, démarche que nous explorerons dans sa dimension méthodologique. Enfin, nous analyserons la capacité applicative et la démarche et nous en brosserons enfin un tableau de perspectives.

\section{Cadre théorique}

\subsection{Introduction}

Le cadre théorique développé dans ce paragraphe a pour finalité principale d'illustrer le cheminement paradoxal d'une démarche systémique, la dynamique des systèmes, dont le destin se révéla fort différent de ce que son initiateur avait imaginé. Conçue au départ pour être une méthode d'analyse de processus de gestion, la démarche s'est en effet illustrée surtout dans des domaines autres que celui du management industriel, et à partir de travaux réalisés par l'initiateur lui-même : analyse de systèmes urbains, modèles du monde, modèle de l'économie américaine ...). De ce fait, le lecteur va (re)-découvrir que le potentiel d'origine de la démarche peut être (et a été depuis quelques décennies) formalisé (par un groupe de chercheurs en organisation) en une méthode pratique de contrôle des processus appliquée au management de l'imprévisible. Il n'est pas moins paradoxal que cet 
imprévisible, largement étudié il y a un siècle dans les systèmes chaotiques, soit depuis peu replacé sous le feu des projecteurs [TAL 10], au travers notamment des systèmes dynamiques non linéaires [STA 13].

\subsection{Systémique et cybernétique}

Dès 1948, le mathématicien américain Norbert Wiener a exprimé les bases d'une science du contrôle des systèmes, vivants ou non vivants, qu'il a appelé la "cybernétique". Etymologiquement, ce mot vient du mot grec "Kubernêsis" désignant "l'action de piloter un navire". Cette nouvelle science du contrôle des systèmes a été évaluée comme tellement puissante, que le gouvernement américain aurait voulu la garder confidentielle. Norbert Wiener s'y est opposé, mais elle est restée dans un cercle de spécialistes. Norbert Wiener prévoit la fin du travail humain remplacé par des machines intelligentes [WIE 52]. Il recommande de préparer une évolution "post-industrielle" des structures de la société, dans laquelle l'homme pourrait enfin être libéré du travail. Faute de prévoir cette évolution sociétale, on serait conduit à un développement sans précédent du chômage et de l'exclusion sociale, pouvant à terme conduire à l'effacement progressif de la démocratie. Au cours des années 50, plusieurs scientifiques ont étudié et renforcé les concepts de la systémique et de la cybernétique appliquées au monde économique et social. Plus tard, Louis Couffignal donne la définition célèbre : "La cybernétique est l'art de l'efficacité de l'action" [COU 63]. A un niveau plus global, les tableaux de bord prospectifs ont aussi pour objectif de rendre efficace l'action. Les concepts de la cybernétique et de la systémique se sont précisés, en particulier, avec les travaux de William Ross Ashby [ASH 56], Joël de Rosnay [ROS 75], Jean Louis Le Moigne [MOI 84].

Notre éducation nous a donné une formation analytique, qui vise à décomposer la représentation des situations complexes jusqu'à les rendre accessibles à l'esprit.

La pensée systémique introduit un changement de paradigme, car elle s'attache à une vision d'ensemble d'un système et de ses sous-ensembles, les uns et les autres dotés d'objectifs, aux liens de causalité entre ses éléments et aux boucles de rétroactions, ou "feed-back", pour réguler le fonctionnement, les interactions, positives et négatives entre ses composantes, et sa régulation dans le temps. C'est un raisonnement qui est de nature dynamique par l'introduction de la dimension du temps, et adaptatif par la prise en compte des boucles de rétroactions et des processus bottom-up. La représentation systémique des organisations se fait en décrivant le fonctionnement des flux économiques en jeu, leurs interrelations positives et négatives jalonnées dans le temps. Un enjeu majeur est la mise en évidence des résultats répartis dans le temps et de détecter les éventuels déséquilibres.

Le jeu de plusieurs scénarios des futurs possibles, dans différentes hypothèses de développement fait apparaître les enjeux et les déséquilibres possibles sur de longues périodes. On fait apparaître, par exemple, l'intérêt d'obtenir moins de résultats dans un domaine pour optimiser l'ensemble, ou moins de résultats immédiats pour améliorer ceux du futur, voire assurer la survie de l'organisation. 


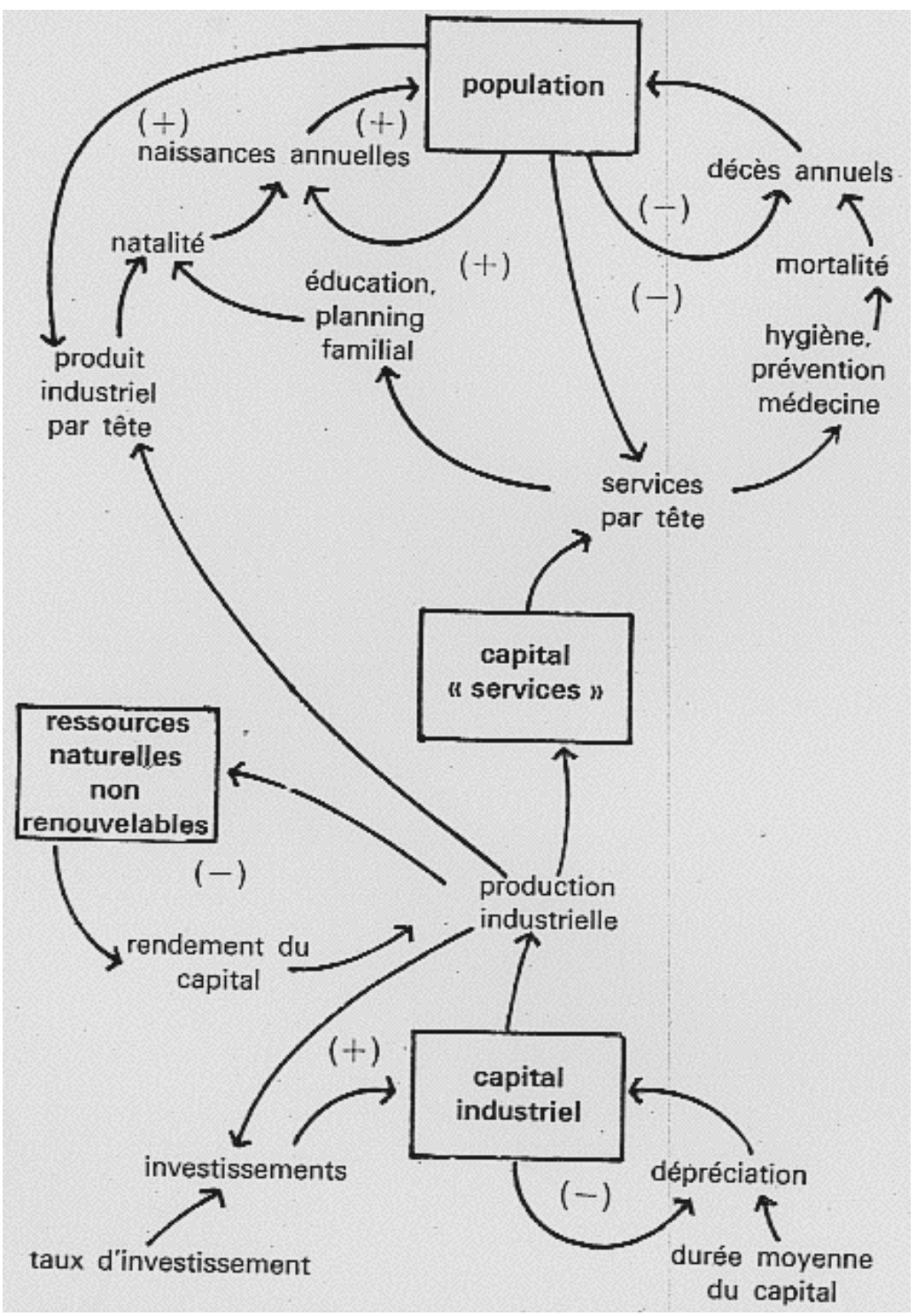

Figure 1. Exemple de représentation de flux et de leurs interrelations (Source : [MEA 72], cité dans [JAN 03])

Cette représentation permet de mettre met en évidence les nécessaires interrelations à établir entre les composantes du système, pour rendre optimum son fonctionnement : le résultat de l'ensemble n'est pas obtenu en maximisant les résultats partiels, mais en optimisant l'ensemble des résultats. La formulation de la composition des résultats est une base de réflexion, de décisions expliquées et concertées. Elle sert ensuite de référentiel pour le suivi et la régulation dans le temps, en vue d'atteindre ou d'ajuster les objectifs fixés.

L'un des apports majeurs de la dynamique des systèmes est de pouvoir projeter les effets des événements d'aujourd'hui sur le fonctionnement futur : on peut ainsi détecter de futures déviations et y pallier en disposant d'un choix de solutions d'ajustements d'autant plus large que l'on dispose de délais longs.

D’une façon générale, la systémique considère le monde comme un ensemble de systèmes et soussystèmes dotés d'objectifs, en interaction (faute d'interactions, un système " meurt »), soumis à des "feed-back" (c'est-à-dire des boucles de régulation) et à de processus bottom-up (permettant une meilleure information des décideurs) :

- Les interactions se font sous forme d'échanges de matière, d'énergie, ou d'information. Chaque élément en interaction réagit en changeant d'état, en modifiant son action, en parant les risques apparus par des boucles de feed-back, positives (on amplifie le phénomène) ou négatives (on provoque l'amortissement du phénomène). 
- A l'intérieur d'un système, les interactions entre les sous-systèmes provoquant actions et réactions rendent le système supérieur plus performant.

Globalement, la systémique énonce des principes de référence :

- Tout système ou sous-système doit posséder un but, représenté par un ensemble d'objectifs.

- Les objectifs du système supérieur se traduisent par des objectifs partiels attribués aux soussystèmes.

- Chacun des sous-systèmes, doté de ses objectifs, peut exécuter sa mission de façon autonome, sous la condition de réaliser ses objectifs, ou d'interagir avec d'autres sous-systèmes pour arriver au but de la façon la plus efficace : la proximité des centres actifs et de leurs objectifs procurent souplesse d'adaptation, rapidité de réaction et accroissement des connaissances utiles.

- Chaque système ou sous-système vit par ses interactions avec son environnement, proche ou lointain : si ses échanges viennent à disparaître, soit il meurt, soit il doit retrouver une source d'échanges. C'est par essence le cas de produits devenus obsolètes : les fabricants de règles à calcul ont dû se reconvertir. Mais le facteur temps joue : on ne se reconvertit pas du jour au lendemain - plus tôt on voit venir le futur, plus on dispose de choix de solutions.

\subsection{Les apports du Pr Jay W. Forrester, du MIT}

Dans les années 1960, le Pr Jay W. Forrester, du MIT (Massachussets Institute of Technology) a apporté une forte avancée pratique, en créant un moyen d'étudier les comportements des systèmes économiques avec des modèles de simulation établis sur ordinateur. On est ainsi passé de la théorie qui a l'avantage de structurer la pensée, au moyen pratique de maîtriser les situations complexes, à travers leur représentation sous forme de modèles informatiques représentant la dynamique des systèmes par l'évolution de leurs flux dans le temps et leurs boucles de régulation.

Jay Forrester apportait une formalisation d'un aspect fondamental de la systémique, les boucles de régulation :

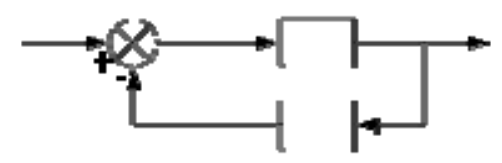

Figure 2. Représentation d'une boucle rétroactive (feed-back) (Source : [FOR 61])

Jay Forrester a appelé son langage de simulation de modèles systémiques DYNAMO, pour Dynamic model et ses publications ont été par la suite toujours faites sous le signe de la dynamique : « lndustrial dynamics » pour la modélisation dans des contextes industriels [FOR 61], appliquée en particulier à la modélisation des transports aux Etats-Unis; «Urban Dynamics » pour l'étude des villes [FOR 69]; « World dynamics » pour une modélisation du monde [FOR 73].

L'ensemble de ses travaux avec leurs applications variées, de l'industrie à l'économie mondiale, se concrétise dans une méthode : la «Dynamique des systèmes». Par sa méthode de simulation de scénarios, J.W. Forrester donne en effet la possibilité de montrer le fonctionnement dynamique des systèmes dans le temps.

Un groupe des élèves du professeur Forrester, animé par Dennis Meadows, a appliqué la méthode à l'économie mondiale [MEA 72], écrit à la demande du Club de Rome, présente une modélisation des interactions complexes dans les sphères économique, démographique et environnementale au niveau de l'économie mondiale. Cette étude a mis en évidence le caractère exponentiel (et insoutenable) des phénomènes économiques soumis à la loi des évolutions dans le temps sous forme de coefficients 
multiplicateurs (chaque année, on vend $\mathrm{x} \%$ de plus, la population augmente de $\mathrm{y} \%, \ldots$ ) : la courbe se « casse » avant longtemps.

\subsection{La dynamique des systèmes}

La dynamique des systèmes peut être définie comme une méthode d'aide à l'intelligence des systèmes complexes [PAU 98]. Utilisée initialement dans le domaine de l'industrie, puis des sciences sociales, ses champs d'application sont devenus extrêmement nombreux et variés [KAR 04], [STE 00], [THI 98]. La dynamique des systèmes peut être définie comme une méthode d'aide à l'intelligence des systèmes complexes (Karsky et Paulré, 1998). Utilisée initialement dans le domaine de l'industrie, puis des sciences sociales, ses champs d'application sont devenus extrêmement nombreux et variés. Alors qu'elle s'est largement développée dans la plupart des régions du monde : Amérique du nord et latine, Europe de l'ouest et de l'est, Asie ; elle est restée à l'état de balbutiements en France. Pourtant, parmi d'autres techniques de modélisation des systèmes complexes, elle permet de créer des modèles de simulation conçus comme de véritables outils pédagogiques. Alors qu'elle s'est largement développée dans la plupart des régions du monde : Amérique du nord et latine, Europe de l'ouest et de l'est, Asie ; elle est restée peu développée en France. Pourtant, parmi d'autres techniques de modélisation des systèmes complexes, elle permet de créer des modèles de simulation conçus comme de véritables outils pédagogiques.

\subsubsection{Point de vue conceptuel}

La dynamique des systèmes est issue de ce que Jay W. Forrester baptisa initialement, à la fin des années cinquante, la «dynamique industrielle». Il est intéressant d'observer que le destin de cette démarche se révèle finalement sensiblement différent de ce que son initiateur avait imaginé. Conçue au départ pour être une méthode d'analyse de processus de gestion, la démarche s'est illustrée en effet surtout dans des domaines autres que celui du management industriel, et à partir de travaux réalisés par Forrester lui-même: analyse de systèmes urbains, modèles du monde, modèle de l'économie américaine ...). Par ailleurs, elle a le plus souvent été utilisée afin d'étudier les propriétés dynamiques de modèles ou de faits " stylisés» sans que la représentation réaliste ou validée d'un phénomène concret, la préoccupation d'une décision opérationnelle, d'une intervention ou encore la réalisation d'un objectif quantitatif précis constituent toujours le contexte ou la motivation déterminante.

Il est cependant clair que, techniquement, rien ne s'oppose à ce que l'on utilise les outils de représentation ou de modélisation de la dynamique des systèmes pour étudier des systèmes d'une autre nature que sociale et/ou économique (par exemple, un modèle d'analyse du développement du cancer). La syntaxe des systèmes dynamiques (celle des systèmes d'équations différentielles non linéaires. non homogènes et non stationnaires) est a priori neutre, c'est-à-dire indépendante de la nature du domaine auquel on souhaite l'appliquer. Mais ce qui caractérisera la démarche de la dynamique des systèmes (par opposition à une démarche consistant uniquement à appliquer un formalisme mathématique), c'est l'interprétation en termes de contre-réactions et de transmission d'information jointe à des effets, voire des boucles purement matérielles. La spécificité de la dynamique des systèmes se situe ainsi, pour une bonne part, au niveau de sa sémantique. Le génie de Forrester avait en effet consisté à lier étroitement syntaxe et sémantique : l'écriture des équations des modèles, en termes de dérivées, d'intégration et de relations fonctionnelles, était censée reproduire la «structure» de la réalité désormais interprétée en termes de liaisons stocks-flux et de relations d'informations. Le modèle et la sémantique de représentation d'un système constituaient, ipso facto, le programme par lequel il était simulé. Un autre élément de différenciation important de la DS tient au caractère continu des variables prises en compte. Cette démarche n'avait pas été conçue pour étudier des systèmes discrets. Il s'agissait, initialement, d'étudier des systèmes dans lesquels les flux sont continus et dont les intensités s'expriment en termes de moyennes, mais avec l'objectif d'étudier les changements de régimes. 
Ce qui est important, du point de vue de l'outil, c'est le va-et-vient permanent entre la structure causale du système, qui fonde sa dynamique, et les évolutions que la simulation fait apparaître. Un système est compris lorsque l'on est capable d'expliquer tous les aspects et toutes les manifestations de sa dynamique par une certaine structure des boucles, les interrelations (les polarités, les gains. les transferts de dominance ...) et les interrelations entre les éléments, les transformations des comportements, etc. La dynamique des systèmes est donc un ensemble d'outils qui servent de support à un travail de compréhension des systèmes complexes, c'est-à-dire de systèmes dont les dynamiques ne sont pas triviales et qui connaissent, le plus souvent. des changements de régime ou des transformations de leurs caractéristiques dynamiques. Dans ces conditions, on comprend que si les fonctions de comportement sont importantes, ce sont surtout les interactions et les caractéristiques structurelles du système qui constituent l'enjeu essentiel du travail d'analyse ou de modélisation.

La thèse centrale de Forrester était que la structure du système (en un sens très précis qu'il définissait à partir des relations d'information, des délais, des points d'amplification) était responsable de sa dynamique. Si cette dynamique avait des propriétés non désirées, il convenait alors d'intervenir en modifiant la structure ou les règles de comportement. On part du principe, scientifiquement d'abord, que l'on peut mettre en correspondance la structure du système et sa dynamique et de l'idée, pragmatique ensuite, que l'on peut modifier la structure de sorte que le fonctionnement soit plus satisfaisant.

\subsubsection{Construction du modèle}

Les modèles permettant d'appréhender la dynamique d'un système se fondent sur les concepts d'interaction, de rétroaction et de complexité. La conception d'un modèle de dynamique de système de type forrestérien consiste tout d'abord à définir la frontière entre le système et son environnement. Les transformations du système résultent, en effet, de modifications plus ou moins contrôlées que subissent les intrants provenant d'autres systèmes ou de l'environnement. Ces transformations produisent, à leur tour, des extrants qu'absorbe l'environnement en général. La construction d'un modèle forrestérien démarre par la réalisation d'un diagramme sagittal. Ce diagramme représente les différents éléments qui composent le système en terme de stocks et de flux, et précisent les relations établies entre les différentes variables du système modélisé. C'est ce qu'on appelle le langage Dynamo. Les éléments de base du modèle sont :

- Les variables de niveau ou d'état. Elles fonctionnent comme des accumulateurs. Ainsi, les variables d'état représentent des stocks dont la quantité varie à travers le temps en fonction des flux d'entrée et de sortie qui les alimentent et les vident. Les stocks sont utilisés pour représenter tant des accumulations matérielles (l'eau, les individus) qu'immatérielles (la connaissance). La valeur de ces stocks renseigne sur l'état du système à chaque instant $t$. A chaque instant $t$, un stock représente l'accumulation passée des flux d'entrées et de sorties.

- Les flux transitent par le réservoir et en modifient donc l'état. Ils déterminent les variations dans les différents niveaux du système. Les vannes contrôlent les débits des différents flux. Chaque vanne peut être considérée comme un centre de décision, recevant des informations et les transformant en actions. En l'absence de flux, aucun changement dans la magnitude des stocks n'est possible.

- Les variables auxiliaires apparaissent dans le canal d'information. Elles peuvent être une constante, ou une fonction tabulée en fonction du temps t ou d'une variable quelconque. Ces variables auxiliaires sont très utiles pour intégrer de l'information qualitative et des délais dans les modèles. Elles permettent également de coupler des flux de nature différente, par exemple un flux d'automobiles et un flux d'hommes.

- Le canal d'information permet de connecter entre elles les variables du système et de simuler les rétroactions. 
Les relations entre les variables du système sont formulées mathématiquement à partir par exemple de lois statistiques, de règles logiques (if then else). Des données quantitatives et qualitatives peuvent ainsi être intégrées au modèle. Il est difficile de dissocier cette approche de l'outil informatique permettant sa mise en œuvre.

\subsection{Autres approches basées sur la dynamique des systèmes}

Quelques applications de modélisations en dynamique de systèmes ont été réalisées en France par des géographes. On peut citer le modèle AMORAL - " Analyse et Modélisation Rurale des Alpes » [CHA 84], le modèle d'un système spatial en zone frontalière franco-suisse [CHE 97], le modèle de catastrophe urbaine associant aléa, vulnérabilité et effets de dominos [PRO 05]. D'autres applications ont été réalisées dans le domaine industriel, par exemple une modélisation d'étude de risque dans des usines de type Seveso [GAR 16] ou dans le domaine de périurbanisation [GAC 08]. Dans cette dernière application, un modèle « d'attractivité de commune » a été élaboré sous forme de diagramme causal réciproques entre une cinquantaine d'éléments liés aux écoles, aux infrastructures, aux emplois, à la demande et à l'offre de logements, au niveau moyen des revenus, à la fiscalité locale, aux dotations de l'Etat, à la perception de la qualité de vie..., sur la base de l'analyse des interactions entre jeux d'acteurs situés à différents niveaux territoriaux et institutionnels.

Les travaux correspondants, développés maintenant dans le monde entier, concernent tous les domaines pour lesquels le temps est une variable essentielle. Citons, entre autres, les domaines suivants en insistant sur ceux qui intéressent plus particulièrement les ingénieurs :

- Stratégie et politique nationale et internationale.

- Finance, marketing, management.

- Environnement, écologie, problèmes urbains, transports.

- Relations humaines (psychosociologie); biologie, médecine.

En effet, cette démarche s'adresse :

- Tout d'abord aux personnes qui travaillent dans les très nombreux domaines où le temps est une variable essentielle, et qui rejettent, en la considérant comme dangereuse, l'attitude « y'a qu'a ».

- À toutes celles qu'intéresse le changement, qu'intrigue l'évolution des structures et des évènements environnants, qui désirent comprendre le pourquoi et le comment de certaines évolutions.

- Donc à quiconque veut analyser et comprendre le comportement dynamique de structures complexes, quelle que soit leur nature (on sait que cette démarche est depuis très longtemps employée, sous d'autres noms ou sous un nom presque semblable, par les scientifiques, les ingénieurs, pour analyser, simuler, améliorer le fonctionnement de systèmes physiques et technologiques : physique nucléaire, aéronautique, construction de centrales, d'usines, de plates-formes pétrolières, etc. C'est avant tout son extension aux systèmes qualitatifs, dits « flous », qui nous intéresse).

- Aux personnes qui désirent mettre en évidence les facteurs structurels agissants d'un système, au-delà d'explications purement conjoncturelles des phénomènes.

Beaucoup d'applications s'appuient sur Stella Research ${ }^{\circledR}$, qui est l'un des logiciels les plus répandus pour modéliser et simuler les systèmes complexes selon les principes de formalisation forrestérienne de stocks et de flux. Ce logiciel comprend deux volets : un module graphique qui sert à construire le modèle puis à présenter les résultats sous forme de courbes, un module mathématique qui est un ensemble d'équations différentielles définies à partir du module graphique. Des applications ont été 
développées en contexte universitaire (Grenoble, Montpellier, ...) dans des modélisations en sciences de la vie et de la Terre (modèle de la température de la Terre, modèle simple du cycle du carbone, modèle de régulation de la glycémie, modèle du cycle de l'eau, ...

«Stella Research ${ }^{\circledR}$ est un logiciel qui permet de modéliser des systèmes, de regarder leur évolution, d'agir sur certains paramètres pour tester différents scénarios. Il permet d'utiliser une approche semblable pour des objets a priori très différents [...] Ce logiciel permet de construire des diagrammes " stocks et flux ". Les stocks sont des sortes de réservoirs, d'accumulateurs qui se remplissent et se vident. Les flux sont des robinets, des connexions qui laissent entrer ou sortir les éléments des stocks. On peut y ajouter des connecteurs qui indiquent de quoi dépendent les flux. A l'aide de quelques équations simples on peut rendre ce modèle quantitatif : il calcule alors l'évolution du système à partir des conditions initiales, et à travers les perturbations que l'on peut lui faire subir. » (http://www.acgrenoble.fr/svt/SITE/portail_tice/cran04/ateliers/atelier14/cord.htm).

Nous observons ainsi bon nombre d'applications de modélisation fondée sur la dynamique des systèmes, principalement par des universitaires, à des fins didactiques d'explicitation, dans divers domaines des sciences de la matière. En revanche, en l'état de nos connaissances après une enquête approfondie, nous n'avons mis à jour aucune trace d'une autre formalisation que celle que nous allons présenter au paragraphe suivant (principalement au $\S 3.4$ et au $\S 3.5$ ) en application pour le management de l'imprévisible dans les organisations.

\section{Adossement méthodologique}

\subsection{Modélisation et simulation}

\subsubsection{Importance de la simulation}

La modélisation instancie un modèle selon un scénario réalisant le cadencement des jeux de paramètres à explorer [SAU 01]. Le modèle a un rôle explicatif en ce sens qu'il doit aider à comprendre le fonctionnement d'un système au du temps, en reproduisant les mécanismes sous-jacents à des évolutions parfopis complexes et apparaissant dans certains cas comme contre-intuitives (en présence de multiples boucles d'amplification ou de régulation, d'effets retardés et, plus généralement, de relations éminemment non linéaires). Le modèle sert également à partager une connaissance et une compréhension communes entre tous les acteurs, en tant que support d'aide à la réflexion, à l'analyse et la prise de décision : il permet de tester l'effet sur le système de perturbations paramétrées et, par oie de conséquence de mettre au jour des leviers d'action [SAU 01].

\subsubsection{Primauté de la simulation variationnelle}

Dans toute approche par modèle, il est impératif d'élaborer une modélisation réaliste, qui, ellemême va instrumenter une simulation variationnelle, au sens où chaque paramètre va, individuellement, être définie dans une plage de variation. Le plan des simulations à mener va cadencer l'exploration des paramètres à fort impact selon un jeu dynamique d'hypothèses d'affectation de valeur aux paramètres, jeu appelé scénario [SAU 01].

PRINCIPE DE LA SIMULATION VARIATIONNELLE : Une modélisation fondée sur la compréhension du système étudié et instanciée par des scénarios variationnels

Un raffinement supplémentaire de la simulation variationnelle est constitué par le principe dynamique du scénario inhérent à la nature des flux. Il est très important de faire la différence entre la simulation fine adossée à une modélisation de chaîne opérationnelle en place nourrie par des paramètres réalistes actuels ou futurs et la démarche des prospectivistes qui imaginent un certain 
nombre d'hypothèses dans le futur et qui en induisent des résultats à un horizon à quelques dizaines d'années, mais sans savoir ce qui se passe entretemps, sans explorer les perturbations intermédiaires.

«Les modèles de dynamique des systèmes ont parfois été considérés comme des outils de prévision, à tort. Leur vocation est avant tout explicative et pédagogique, les boucles de rétroaction, les délais, les relations non linéaires sont autant d'éléments qui peuvent conduire à des évolutions complexes. L'outil de simulation est là pour aider à en comprendre le fonctionnement, à tester des scénarios pour des futurs possibles (prospective notamment), des mesures pouvant aider à enrayer des mécaniques pernicieuses, etc. Il peut être considéré comme un outil d'aide à la gouvernance des systèmes, un support d'aide à la décision, interdisciplinaire. » (Les modèles de dynamique des systèmes : des outils pédagogiques pour une aide à la gouvernance des systèmes, Valérie GACOGNE, COMPLEXIO, 2009)

De plus, la dynamique des systèmes est, selon son inventeur Jay Forrester, "un mode d'étude du comportement [...], permettant de montrer comment des politiques, des décisions, des structures et des délais sont en interrelation pour influencer la croissance et la stabilité. " [FOR 61]. Cette démarche qui repose sur l'analyse des systèmes répond à une logique notablement différente de celle qui fonde l'économétrie - dite de "l'induction statistique" - dont le principe fondateur est de " déterminer une procédure qui, à partir des seules observations dont dispose l'économètre, permette de passer de l'espace échantillon à l'espace des structures " . On substitue en fait à une démarche relativement synthétique visant à rendre compte de relations complexes à l'aide d'un nombre réduit de formules mathématiques "ajustées" elles-mêmes souvent complexes, à une démarche visant à décrire les relations causales du système. D'un côté, l'économétrie, pour laquelle la disponibilité de séries statistiques et le travail d'identification et d'ajustement sont à la base de la vérification d'hypothèses théoriques. De l'autre, la dynamique des systèmes, qui repose essentiellement sur la compréhension des interrelations et des influences entre éléments du système. D'où l'importance de la connaissance descriptive du système. Cette différence d'approche donne naissance à des modèles de nature assez différente. La dynamique des systèmes met en scène plus d'interrelations, plus de boucles de rétroactions, plus de mécanismes d'adaptation et de rétroaction temporelle. Bref, à des relations essentiellement linéaires construites pour obtenir in fine une image à un certain horizon, on substitue des relations non-linéaires, permettant de décrire l'évolution du comportement du système pendant un certain temps. La différence est essentielle, tant dans la conception d'un modèle que pour son usage.

La dynamique des systèmes nécessite sans doute un travail plus approfondi de compréhension du système. L'analyse causale en est la trace indéniable. Et sa transposition opérationnelle en équations concrètement en un diagramme Stella ${ }^{\circledR}$ - nécessite un approfondissement supplémentaire.

\subsection{Contexte de développement de l'approche CADEV}

\subsubsection{Des années 1950 aux années 1980}

Dans les années 1950 à 1970, les organisateurs avaient pour rôle de mettre en place des modes de travail et de communication entre services, des circulations de produits et d'informations. Parmi leurs buts, ils devaient prendre en compte le caractère aléatoire des fonctionnements économiques et les risques matériels ou économiques, permettre un travail équilibré pour le personnel, dans la limite de fatigues acceptables (... on ne se jetait pas par la fenêtre), avec les moyens nécessaires pour assurer la sécurité, permettre un service de qualité aux clients, et assurer la rentabilité de l'entreprise dans le temps. Les organisateurs étaient confrontés à la complexité de fonctionnement des ensembles économiques, avec leurs nombreuses interrelations et interactions, internes et externes. Ils possédaient des méthodes éprouvées, mais où les interactions dans le temps entre les flux et les fonctions de décision étaient mal représentées. Ils ont donc été très directement intéressés par les approches systémiques et dynamiques des flux économiques, qui ont émergé pratiquement à cette époque. 
Dans les années 1960, les avancées du Pr Jay W. Forrester au MIT et sa méthode de représentation des activités économiques [FOR 61] ont fait l'objet d'analyses approfondies, destinées à la compréhension de la démarche et des modèles proposés, au sein d'un groupe de recherche à l'IESTO (Institut d'Etudes Supérieures des Techniques d'Organisation), un des Instituts du CNAM (Conservatoire National des Arts et Métiers) : sa méthode de "Dynamique des systèmes" apportait un complément à leur propre démarche dans leur métier d'organisateurs : les méthodes d'analyse et de conception de nouvelles organisations permettaient de sortir des moyens limités de représentation du fonctionnement des organisations et de leurs interactions jusqu'alors utilisées. Ce groupe de recherches de l'IESTO s'est donc attaché à la compréhension des concepts sous-tendus par la méthode, proposée par Forrester, de modélisation de l'organisation sous forme de flux dynamiques. Il a ainsi formalisé une méthode pratique pour représenter et organiser sous forme systémique et dynamique les quatre flux économiques désignés par Forrester : Produits, Ressources (matérielles et humaines), Activités, Argent.

\subsubsection{Depuis les années 2000}

Ces dernières années, le besoin de réagir mieux et plus vite, dans des conditions économiques de plus en plus tendues, a conduit à de nouvelles réflexions sur les méthodes systémiques. Leur conception interactive et dynamique était adaptée aux changements profonds des situations économiques avec des interrelations mondiales, à l'accélération des changements, et à la nécessité d'évaluer plus que jamais des situations dans le temps. Dans les réponses aux besoins de connaître les prévisions du futur, les offres de la Business Intelligence semblent avoir brouillé les cartes. Pendant plusieurs années, les prévisions du futur ont été déduites du passé. Ensuite, devant les évolutions très rapides des situations dans le temps, les prévisions se sont faites, non plus en se référant au passé, mais au présent. Finalement, au cours de l'année 2008, les sociétés de Business Intelligence ont été rachetées, et leurs produits intégrés dans les systèmes d'information.

Face aux offres de Business Intelligence se référant au passé ou au présent, la société CADEV, société de conseil et de formation, a conçu une plateforme de simulation systémique et événementielle du fonctionnement des organisations, primée par le Ministère de la Recherche comme produit «IT» innovant.

La société CADEV Interactive a été créée pour développer cette plateforme qui comporte trois volets.

\subsection{Principe de simulation : des hypothèses du futur à deux niveaux}

La méthode permet de simuler des hypothèses du futur et réunit, dans une structure intégrée, deux niveaux de fonctionnement : les prévisions stratégiques pour le futur et les prévisions de pilotage du réel.

La simulation des scénarios du futur dans une vision stratégique fournit les résultats à attendre et les risques encourus, période par période. Le jeu de plusieurs hypothèses fournit une base de concertation et de décision. Les futurs possibles et leurs conséquences sont imaginés - les politiques d'innovation et les interactions avec les organisations externes peuvent être testées. La simulation de scénarios alternatifs fournit une mesure des changements, des évolutions techniques et sociales induites, des risques et de l'impact de leur gravité. Les résultats anticipés permettent une prise en compte des risques, une concertation, et conduisent aux choix stratégiques et à la mise en place des moyens nécessaires au fonctionnement décidé et à son contrôle. La simulation des scénarios du futur dans une vision de pilotage du réel conduit à ce que, une fois prise une décision sur le scénario stratégique à retenir, les prévisions deviennent les cibles du fonctionnement réel, et le contrôle de l'atteinte des objectifs doit être permanent. L'idée est de ne pas seulement vérifier si les événements du quotidien vérifient les prévisions stratégiques, mais de simuler leurs effets dans le temps pour savoir ajuster le fonctionnement dans la perspective des objectifs à moyen terme. 
Le positionnement de signaux d'alarmes, tels que les écarts inacceptables, permettra aux décideurs désignés d'être automatiquement informés des déviations anormales, de réagir, de corriger le fonctionnement réel, voire de déclencher un ajustement des plans stratégiques. Les informations sur le fonctionnement réel, lorsqu'elles existent dans le SI, sont intégrées de façon automatique. Les informations qui n'existent pas sont entrées de façon aussi automatique que possible, avec par exemple l'utilisation de codes à barres. Quelle que soit la taille de l'organisation, la puissance des représentations systémiques permet de représenter le fonctionnement des sous-ensembles avec leurs interactions, de mesurer les résultats à chaque niveau de responsabilité pour assurer les boucles de régulation et l'efficacité. Les flux sont simulés par la gestion des "événements" représentant le fonctionnement. Les événements sont jalonnés dans le temps, sur des périodes plus ou moins longues suivant la perspective de temps analysée : l'année pour les projections prospectives ou les évaluations stratégiques, le mois, la semaine ou le jour pour le suivi du pilotage. Les fonctions de liaisons assurent la coordination entre les flux. Dans les phases d'études prévisionnelles, on peut sans risque "jouer" plusieurs hypothèses de scénarios, évaluer les gains et les risques de chacun d'eux. Dans les phases de pilotage, les fonctions de contrôles vérifient la conformité entre les prévisions et le réel, tel qu'il existe ou tel qu'il s'annonce inéluctablement pour le futur, eu égard aux événements vécus (un retard au bureau d'études se répercutera sur le délai final : cela se mesure... ).

\subsection{Genèse de la méthode CADEV}

Après la dispersion du groupe de recherches de l'IESTO, ses travaux ont été transposés dans le cadre d'un cabinet de conseil en organisation, CADEV. Les conseils se sont attachés à reprendre l'étude des travaux de Forrester, pour aboutir cette fois à une utilisation pratique dans leurs missions d'organisation. Ils ont pu mettre en pratique cette nouvelle démarche dynamique dans leurs analyses et dans la construction de nouvelles solutions d'organisation.

La mise en évidence simple des interactions entre services et fonctions s'avérait particulièrement fructueuse dans la dynamique des interrelations, des choix faits en groupes de projets et pour l'optimisation de la réalité quotidienne. La Fédération des Industries Mécaniques (FIM) a fait un appel d'offres pour mettre au point un Guide Méthodologique destiné à ses adhérents soucieux de réussir l'implantation d'une GPAO (Gestion de la Production Assistée par Ordinateur) dans leurs entreprises. Un groupe de chefs d'entreprise avait été réuni à cet effet. CADEV a été retenu par ce groupe grâce à l'apport de la méthode de Forrester. Le travail du groupe a abouti à la rédaction d'un Guide [FIM 86].

Aux Etats-Unis, cette méthode est largement utilisée, en particulier dans l'industrie (Boeing par exemple), dans des études de développement durable pour modéliser les relations entre les ensembles, les sous-ensembles et l'environnement.

\subsection{Approche CADEV : une démarche dynamique et participative}

En France, CADEV a créé une version opérationnelle de cette méthode pour modéliser et optimiser les processus économiques en groupes de projet, en coopération avec les personnes impliquées dans le fonctionnement et avec les conseils d'experts.

LE PRINCIPE DE L'APPROCHE : Le principe consiste à coopérer pour optimiser et dynamiser l'activité : il s'agit d'étudier et d'organiser ensemble les flux physiques des activités économiques et les flux d'information qui les irriguent, en application de la dynamique des systèmes économiques élaborée par le professeur Forrester.

De ce principe découle une approche constituant un outil puissant, simple à utiliser, pour l'organisation des activités et de leurs interrelations, les prévisions stratégiques, le pilotage dynamique du réel et la maîtrise des risques.

La finalité de l'approche est donc de maîtriser : 
- La complexité des systèmes économiques interconnectés.

- La dynamique des interactions jalonnées dans le temps.

- Les déséquilibres identifiés, immédiats et futurs.

- Le caractère aléatoire et risqué du fonctionnement économique et social.

en décrivant, améliorant et optimisant le fonctionnement interactif de quatre flux économiques (Flux Produits, Flux Ressources, Flux Activités, Flux Finances), en coopération avec tous les acteurs impliqués (acteurs de tout rang hiérarchique et experts). Les interactions des événements sur les flux économiques sont jalonnées dans le temps. Les déséquilibres, immédiats ou futurs, sont identifiés, supprimés ou maîtrisés. Les flux d'informations irriguent les flux physiques et ils déclenchent feedbacks et alarmes pour maitriser les anomalies du fonctionnement.

L'organisation des flux économiques s'appuie sur une méthode graphique décrivant les flux et leurs interactions. Ces flux ont été définis par Forrester comme :

1. Le savoir-faire et les produits.

2. Les ressources (équipements, énergies, travailleurs).

3. Les activités.

4. Les finances.

Il existe des liens entre les quatre flux, qui les rendent "vivants" par leurs interactions (en systémique, on dit qu'un système privé d'interactions meurt) et permettent d'arriver ensemble aux résultats attendus, qui constituent des cibles à évaluer. Chaque flux est jalonné par des "événements" qui transforment les flux. L'analyse des événements a montré qu'ils appartiennent à un nombre de types limité (une soixantaine de " fonctions de transformation »). Les flux, en se déroulant, impliquent une prise en compte du temps, facteur fondamental dans l'évaluation des résultats : il n'est pas indifférent de constater des gains ou des ruptures dans leur répartition temporelle ou dans leur concomitance avec d'autres résultats.

La méthode propose le langage graphique simple de six symboles, d'après Forrester : Opération, Contrôle, Stockage, Décision, Source [entrée], Puits [destination]. Il constitue un langage commun aux décideurs, acteurs, concepteurs, organisateurs, responsables de projet : simple, il favorise le travail en commun entre personnes de formations, de rangs hiérarchiques différents. La description de chaque activité utilise un langage simple, avec six symboles graphiques qui se décrivent avec soixante mots du langage courant, par exemple : Recevoir - Envoyer - Afficher - Lire - Écrire - Calculer - Comparer ...., suffisants pour décrire le fonctionnement de toute organisation, même complexe. On y a simplement introduit deux symboles nouveaux, pour tenir spécialement compte de la gestion des "flux" économiques proposée par le Pr Jay Forrester :

- un rectangle remplace le classique triangle du stockage pour représenter un « réservoir», marquant l'arrêt d'un flux par un stockage.

- une vanne enrichit la représentation du système d'information et représente la fonction de décision, et son ouverture vers les décideurs de chacun des niveaux concernés.

La modélisation du déroulement des processus se décrit dans des graphes représentant les suites d'opérations et les relations entre les quatre flux économiques : Produits, Ressources, Activités, Argent. Un automate transforme les graphes des modèles obtenus en logiciels, en supprimant la phase de programmation. Le logiciel, connecté aux processus réels et à une base de données Big Data, accompagne et contrôle le fonctionnement pratique des organisations économiques de toutes natures et de toutes dimensions, dans deux perspectives : stratégique et opérationnelle. 
Deux perspectives temporelles sont envisagées :

- Pour le futur : afin de prévoir, décider, organiser les activités futures, des scénarios des futurs imaginés font apparaître le comportement futur de l'organisation par période, les résultats à attendre et les risques potentiels, dans différentes hypothèses d'organisation et d'activités à venir. Le jeu de plusieurs scénarios des futurs possibles permet la concertation, les décisions et la mise en place d'une organisation appropriée.

- Pour le vécu : afin de suivre les activités réelles interconnectées et de maîtriser les éventuels déséquilibres, le traitement instantané des données reçues et de leurs impacts sur le fonctionnement des quatre flux économiques, permet de détecter et combattre les éventuels déséquilibres, immédiats ou à venir, et de piloter les activités avec des tableaux de bord et des procédures d'alarmes.

Les domaines d'applications incluent les entreprises, grands groupes ou PMI/PME, les territoires, les organisations économiques. La méthode, utilisée dans le cas de la FIM, est essentiellement généralisable, grâce à sa conception systémique : le langage des systèmes s'applique à la représentation de l'ensemble d'un système de quelque nature qu'il soit, comme à celle d'une partie du système. En effet, il faut choisir le niveau de représentation qui correspond le mieux au but de l'étude que l'on engage, comme on choisit l'échelle d'une carte géographique en fonction de l'usage que l'on veut en faire.

Ainsi, pour les organisations économiques, la représentation, faite sous forme de graphes dynamiques d'ensembles et sous-ensembles en interactions, permet :

- Une étude progressive de l'organisation économique et des traitements des informations Chaque sous-ensemble peut être librement organisé, en respectant deux conditions : atteindre les objectifs qui lui sont fixés dans le cadre des objectifs d'ensemble et communiquer avec les sous-ensembles environnants (sous peine de " mourir », dit la théorie des systèmes !).

- La mise en cohérence de l'ensemble des activités et des rôles, en commençant par les activités qui posent des problèmes - activités souvent mineures, mais maillons faibles et forts perturbateurs du fonctionnement d'ensemble.

- L'optimisation et la simplification du fonctionnement et de son déroulement dans le temps, tout en assurant une gestion des risques (prévention, puis alerte en cas d'émergence dans le réel).

- La définition des articulations entre les activités, et la communication aux acteurs de leurs objectifs.

L'objectif n'est pas de maximiser les résultats du fonctionnement de chaque sous-ensemble, mais d'optimiser la rentabilité et la sécurité de l'ensemble, dans des perspectives de performances, de qualité, de bonnes conditions de travail et de sécurité. 


\subsection{La mise en œuvre de la méthode comporte quatre étapes}

\subsection{1. $1^{\text {ère }}$ étape : Définition du système et de ses interactions avec l'extérieur}

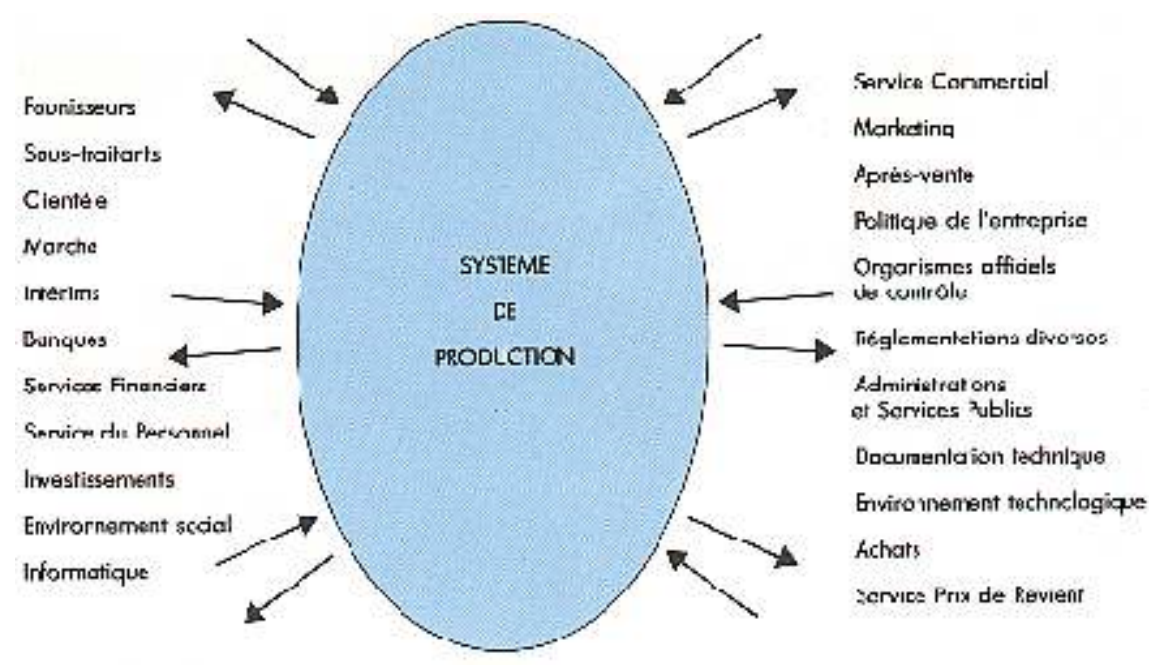

Figure 3. Définition du système et de ses interactions avec l'extérieur (Source : auteur)

La première étape définit les limites du système étudié, les sous-ensembles qui le composent, leurs objectifs respectifs et le type de leurs relations. Les objectifs et les liens précisés, les analyses bénéficient pleinement de l'approche systémique, grâce à la liberté de choisir l'ordre dans lequel on étudiera les sous-systèmes. Il y a en effet toujours des sous-systèmes plus complexes que les autres ou plus sensibles sur le plan des risques - de ce fait ils sont plus longs à analyser, impliquent l'intervention d'experts plus nombreux, ... Cette capacité systémique à traiter chaque sous-ensemble séparément, tout en préservant l'unité de fonctionnement de l'ensemble supérieur, est une source de souplesse et d'efficacité. La phase de définition du système, de ses limites et de ses composants se termine par la désignation des personnes qui participeront aux études de chaque sous-ensemble.

3.6.2. $2^{\text {ème }}$ étape : Définition des quatre flux et de leurs sous-ensembles, avec leurs limites et leurs objectifs (sous-systèmes)

1. Produits - Composants.

2. Ressources, équipements et énergies, dont les Ressources Humaine.

3. Activités.

4. Financier.

Suivant le référentiel de Forrester, le flux d'information "irrigue" les flux économiques : il les accompagne et il se définit naturellement dans la description des activités.

\subsection{3. $3^{\text {ème }}$ étape : Définition du déroulement des flux}

Forrester propose de qualifier les événements qui jalonnent les flux en utilisant six symboles graphiques : 


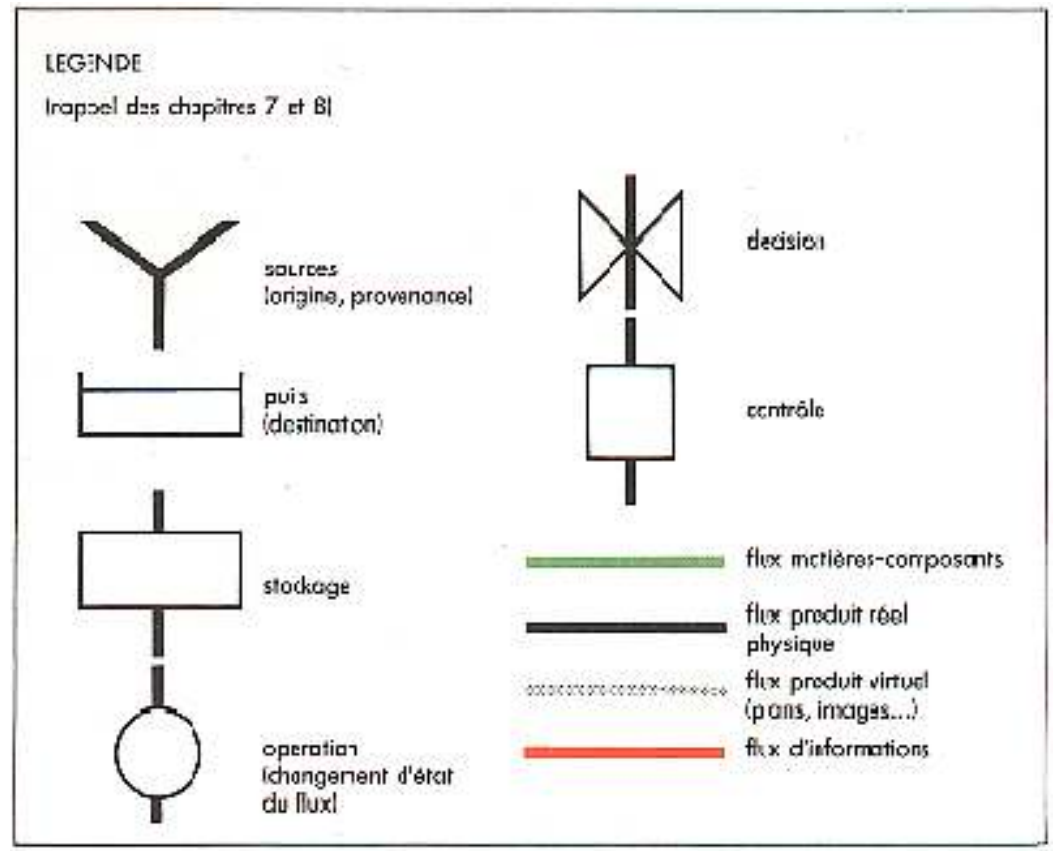

Figure 4. Symbologie de base (Source : [FOR 61])

Ces symboles, simples et peu nombreux, sont appris rapidement par les participants des groupes de travail et permettent de réunir dans des groupes de projet des personnes de formation ou de niveaux de responsabilité différents : ils se comprennent et coopèrent dans la recherche d'une description commune des processus. La description des situations réelles, ou imaginées pour le futur, est d'autant plus exacte et complète que des acteurs impliqués dans le fonctionnement de chacun des flux participent à l'étude.

\subsection{4. $4^{\text {ème }}$ étape : Recherche d'améliorations}

Les améliorations recherchées concernent les conditions de travail et les communications, l'optimisation des résultats et la prise en charge des risques. Inspiré des modèles de représentation de flux de Forrester, la méthode fournit ainsi une méthode d'amélioration du fonctionnement des organisations et de leurs interrelations, et un cadre de gestion prévisionnelle et de pilotage.

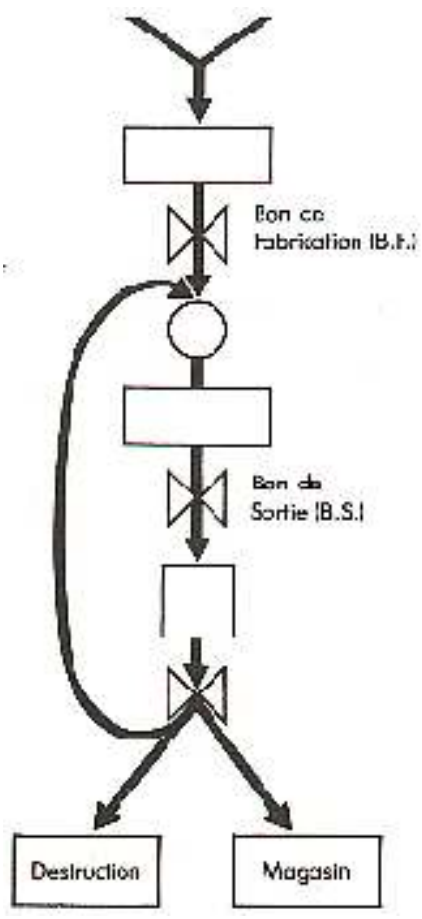

Figure 5. Un flux simple, avec une boucle de régulation (Source : auteur) 


\subsection{Equilibrages des organisations}

Les déséquilibres économiques et sociaux viennent des interactions et des boucles de rétroaction positives dans le fonctionnement des organisations : ces boucles amplifient les phénomènes, en particulier lorsqu'ils sont de type exponentiel. Le rapport du Club de Rome, en 1972, était basé sur l'étude d'hypothèses du développement de l'économie mondiale : le résultat mettait en évidence les risques d'un effondrement économique dans une perspective de quelques décennies, au plus tard en 2100 [MEA 72]. La même étude a été reprise en 2004 avec les données alors connues : 1'effondrement s'annonçait pour 2040... [MEA 04]

Au niveau mondial, la conscience est apparue ces derniers mois, avec la crise, qu'il était nécessaire d'établir des accords entre nations. Des "sommets" réunissent les responsables de plus en plus de nations. En ce qui concerne les déséquilibres macro-économiques, rappelons qu'en 1950 Norbert Wiener a recommandé de préparer une évolution "post-industrielle" des structures de la société, et il prévoyait que, faute de prévoir cette évolution sociétale, on serait conduit à un développement sans précédent du chômage et de l'exclusion sociale, pouvant à terme conduire à l'effacement progressif de la démocratie.

Les événements sociaux de ces derniers mois, tels la fermeture de l'usine Continental à Clairoix s'ajoutant à tant d'autres, montrent la pertinence de cette prévision datant de plus de 50 ans. Pour les organisations économiques, les déséquilibres sont des contraintes tout aussi graves, quoiqu'à une moindre échelle, et sur des perspectives de temps plus courtes. Il est absurde de penser qu'un futur déséquilibré est certes possible, mais qu'on n'y peut rien, donc autant fermer les yeux, ne pas y penser et bien sûr ne rien faire. Les crises du futur sont inscrites dans les événements d'aujourd'hui, de la même façon que nous subissons aujourd'hui les effets des conduites du passé.

Vivant dans un monde aléatoire, et par ailleurs rempli d'interconnexions avec bien d'autres organisations, parfois inattendues, nous ne pouvons pas être entièrement assurés du futur - il existe toujours plusieurs hypothèses des possibles. Du moins faut-il envisager le champ des possibles, les plus probables et les plus extrêmes (c'est à dire entraînant la "mort" de l'organisme). Seule une projection dans le temps des variables de fonctionnement jugées significatives peut mettre en évidence les choix possibles et servir de support de réflexion. Les choix conduisant à terme à la "mort" peuvent être identifiés et combattus. Ensuite viennent les décisions entre plusieurs possibles, en privilégiant les gains à court terme ou les gains cumulés à moyen et long terme. 11 existe évidemment, à l'intérieur de chaque organisme, des intérêts contraires, des luttes d'influence :

- Dans une vue à court terme, les intérêts s'opposent.

- Dans une vision à long terme, les intérêts convergent, en montrant que s'il y a perte pour l'organisme, tout le monde y perdra.

L'exemple des retards de l'A380, et de leurs conséquences, en est une illustration. La Gouvernance est souvent évoquée, mais s'exerce avec difficultés [DEN 16]. Comment décider sans perspectives sur les développements futurs et sur les risques potentiels ? La simulation de scénarios du futur, avec le déroulement dans le temps des événements associés à des hypothèses d'activités, permet de connaître les variations des résultats à attendre, les risques associés et leur gravité, période par période. Comment ensuite contrôler le réel, détecter les anomalies et les évolutions inattendues, ajuster les prévisions et le fonctionnement à des situations nouvelles, envisagées dans les hypothèses d'activités, ou inattendues, si on n'a pas formulé des hypothèses prévisionnelles ? La disposition de tableaux de bord prospectifs apporte une solution, en enregistrant en permanence le réel et en projetant ses conséquences sur le fonctionnement à venir dans le court terme, en comparant les résultats à attendre avec les prévisions stratégiques, avec déclenchement automatique d'alarmes pour signaler les écarts significatifs avec le prévu - si tout va bien, n'en parlons pas. 
On argue souvent de la complexité des situations, du manque de temps pour réfléchir et prévoir, mais on vit au quotidien dans le risque incontrôlé et on passe du temps à refaire sans fin des prévisions du court terme, alors que :

- La systémique apporte une solution de maîtrise de la complexité.

- La cybernétique et ses principes de pilotage apportent une solution de gouvernance.

Dans un monde de déséquilibres qui s'imposent de l'extérieur, le rôle de chaque organisation est de prévoir et gouverner, pour n'être pas elle-même conduite au déséquilibre, et, in fine, à l'échec, voire à sa perte.

\section{Capacité applicative et perspectives}

\subsection{Potentiel applicatif}

Une part importante du potentiel applicatif de la démarche provient du fait que les conseils font faire intervenir le personnel de leurs sociétés clientes en groupe de travail pour le projet, ce qui résout le problème de l'adhésion du personnel puisque la démarche est appliquée avec eux. De plus, en animant les groupes de travail, les conseils organisateurs vont calyser la communication entre représentants de différents métiers, de différents services et donc œuvrer à réduire la distance cognitive entre les membres du personnel.

Pour des raisons de confidentialité, il est bien sûr difficile de donner à voir le déroulement de cas réels de missions d'organisation, de résultats de simulations de chaînes organisationnelles et productives (quelle que soit l'activité produite), de ressenti des participants face au changement introduit, d'impact de l'approche sur l'organisation et sur la transformation que celle-ci peut être amenée à engager. Toutefois, nous pouvons ajouter que, pour le déroulement de la vie réelle de l'entreprise, ce n'est pas seulement la partie industrielle qui intervient, mais également la partie administrative. Notre expérience nous a montré que l'introduction, dans les tâches administratives de gestion, de suivi et de contrôle, ..., d'outils individualisables comme les tableurs augmente la propension des agents à faire les choses en double : chacun se monte un modèle de calcul spécifique à sa propre façon d'envisager sa tâche sans se préoccuper d'un modèle partageable, et ce, d'autant plus facilement que son propre outil n'est pas visible aux autres. Ceci va se traduire par un manque de pensée d'ensemble, dans toutes sortes de travaux routiniers. Dans ce contexte administratif assez opaque, où chacun essaie d'instrumenter son propre maillon avec son propre outil, pour des tâches souvent menées en double, il est donc particulièrement utile de mettre au jour, d'analyser et de simuler les chaînes de production administrative sur la base des quatre flux Produits, Ressources, Activités, Argent. Pour réaliser l'analyse des rouages de l'activité de chacun et en extraire les principes à modéliser, nous avons appris empiriquement que les conseils devaient directement interroger les intéressés (les plus à même de décrire leurs tâches) sans surtout passer par leur supérieur hiérarchique qui, au-delà de comment les choses devraient se passer dans le principe, n'a souvent qu'une vision très incomplète du déroulement des choses dans la réalité quotidienne.

D'un point de vue pragmatique, soulignons que la simulation du chaine opérationnelle productive tenant compte de tous les facteurs environnementaux internes ou externes va se traduire par une appréciation immédiate des conséquences d'un dérèglement ponctuel de cette chaîne, une évaluation en termes de risque et une décision simple de prévention. Par exemple, l'hiver, les intempéries peuvent conduire à des retards de livraison de matières premières ou intermédiaires, immobilisant les ateliers de fabrication. Dans ce cas, quelques soient les objectifs de réduction des immobilisations financières en stocks, il sera peut-être préférable, en hiver, de consentir une augmentation des stocks de sécurité pour réduire les risques de pertes d'activité. De même, la prise en compte du séquencement temporel dans la simulation permet de quantifier l'impact d'un aléa : par exemple, un retard dans la chaîne en amont va 
entrainer des retards de livraisons et le mécontentement des clients que l'on perdra peut-être, des retards d'encaissements conduisant à des déséquilibres financiers, peu appréciés des banquiers, etc...

Ces exemples simples illustrent très clairement l'apport de la démarche appliquant la dynamique des systèmes à la gestion prévisionnelle des aléas dans la vie quotidienne des entreprises et des organisations. Une extension de cette approche consiste à généraliser l'appréhension des aléas à de plus subtiles perturbations peu prévisibles mais à très fort impact. Nassim Taleb appelle «Cygnes noirs » ces changements inattendus de paradigme, d'usage, de produits [TAL 10]. Ces changements subits ont de profonds impacts. Quand ils surviennent, ils sont incontournables et bouleversent l'existant, voire le suppriment : il faut changer de monde et savoir sauter au-dessus de l'abime ... ou y chuter. Imaginer ces cygnes noirs, d'avance, dans des scénarios des futurs possibles de nouvelles technologies, de nouvelles conditions sociales, de nouveaux modes de consommation. Mesurer leurs impacts et mener les actions nécessaires pour s'y préparer, s'y adapter par des transformations profondes, les pallier dans le court terme, doit être l'un des buts de la démarche de «management des futurs » dans les organisations économiques, entreprises, villes, régions, groupements de toutes natures.

\subsection{Perspectives}

Les contextes technologique et économique sont en cours de modification accélérée, les modes de travail et de transport, les modes de consommation et d'achat, et plus généralement les modes de vie, sont influencés par des changements technologiques et sociaux profonds, déjà amorcés mais qui vont s'étendre dans les proches années à venir.

En usine, les robots vont prendre la place de personnes. Par exemple, 4 ouvriers à des postes de travail pénibles vont être remplacés par 4 robots, conduits par un seul opérateur connecté, dans des conditions matérielles meilleures - en principe, mais que sait-on du stress subi ? Que deviennent les trois autres ouvriers ? Que deviennent les entreprises? En France, la création de régions plus vastes et moins nombreuses permet d'envisager une nouvelle gouvernance, la capacité de mener des actions plus puissantes et élargies. Peut-être une économie locale décentralisée, rentable, de meilleure qualité, évitant les coûts et la pollution de transports lointains, paraissant souvent tellement absurdes !

La vie des villes, pourra aussi être remise en cause, en particulier avec des modes d'achats, de consommation et de distribution différents. Que se passera-t-il si les commerces en centre-ville renaissent après avoir presque disparus, désertés au profit des grandes surfaces périphériques ? Que deviendront d'ailleurs les centres commerciaux à leur tour désertés ? Et si les livraisons à domicile se développent, faites par des personnes sous-payées, mais tellement commodes ? Mais comment vivent ces personnes qui sont sous-payées pour un travail souvent dur, pour quel avenir et avec quelle vie sociale?

Par ailleurs, sur le plan industriel, on constate maintenant la disparition subite d'entreprises anciennes, qui ont été prospères et mettent la clé sous la porte - ou sont maintenues artificiellement, en général pour partie seulement. Tout simplement, elles vendent des produits dont les générations nouvelles n'ont aucun besoin! Et qui a donc réfléchi d'avance à cette évolution et à ses conséquences, en particulier sur le plan de l'emploi ? Des modèles alternatifs pourraient être imaginés et testés sans danger par la simulation économique ... avant l'obligation de la mise de la clé sous la porte.

Le changement se prévoit et se prépare, mais il doit être voulu et réfléchi.

Les instances d'économie régionale mènent depuis des années des actions visant à améliorer le fonctionnement des activités dans une vue prospective, en cherchant à optimiser les services rendus à la population et leurs coûts. Il paraitrait positif de créer des liens interactifs avec les nouveaux territoires élargis et de mener des actions coordonnées pour aborder un monde modifié par les 
technologies nouvelles, conduisant à l'abandon d'activités et de produits, avec de nouveaux métiers et leurs impacts sociaux et économiques, avec des déséquilibres entre villes et campagnes.

L'innovation est actuellement encouragée. Elle est un facteur déterminant dans le contexte de changements profonds, technologiques et sociaux que nous vivons. Probablement, par essence, paraitelle difficile à mettre sous forme de modèle. Mais en reprenant les bases de Forrester, on constate que les conditions d'une approche systémique est possible : les quatre flux existent bien - Produits, Ressources, Activités, Argent. Fixer des buts paraitrait adéquat.

Bien sûr, il faut bénéficier du pouvoir de l'imagination des innovateurs, mais de grandes lignes peuvent être déduites de l'observation des évolutions au niveau international et des travaux des chercheurs. Analyser la possibilité, moyennant quelques adaptations, d'appliquer la méthode à des activités liées à l'innovation, montrer que le management de l'innovation pourrait se nourrir aussi de ces flux et d'une prévision de ces flux.

\section{Conclusion}

Le déroulement des opérations dans une entreprise est de plus en plus perturbé par des éléments imprévisibles de leur environnement interne ou externe. Une réponse à cette situation peut être fondée sur la dynamique des systèmes. Celle-ci, initialement conçue pour fournir une méthode d'analyse de processus de gestion, a prospéré surtout dans des domaines autres que celui du management industriel : analyse de systèmes urbains, modèles du monde, modèle de l'économie américaine .... Nous avons vu que, en marge de cet essor académique, le retour à l'esprit initial inspiré par la cybernétique a permis de stimuler la modélisation d'une chaîne d'activité industrielle, sur la base de la description et de l'exploration des propriétés dynamiques de ses modèles. Grâce à la représentation réaliste de ces derniers en termes de dynamiques temporelles de flux irriguant un environnement industriel considéré comme un système non linéaire dynamique réagissant à des perturbations paramétrées à caractère aléatoire, la simulation décrite constitue un puissant moyen d'aide à la décision, tant opérationnelle (au sens du court terme) que stratégique (au sens du long terme).

Les leçons apprises sont liées à l'approche méthodologique à deux niveaux de la problématique traitée, situation tout à fait caractéristique d'une gestion des connaissances inventives [SAU 13] : un niveau d'analyse de la structure de la connaissance inventive déposée dans les connaissances inventives (theoria) et un niveau d'application des résultats de cette analyse à la prise de décision (praxis). Le premier niveau est instrumenté par un outil de simulation modélisant un système dynamique non linéaire et le deuxième niveau est adossé aux sorties de cet outil optimisant la décision : ce qui fonde le succès de l'approche est le va-et-vient permanent entre les deux niveaux, c'est le va-etvient permanent entre la structure causale du système (en particulier les interactions entre les soussystèmes), qui fonde sa dynamique $\left(1^{\text {er }}\right.$ niveau) et les évolutions $\left({ }^{\text {ème }}\right.$ niveau $)$ que l'instanciation de la simulation selon un plan d'expérimentation raisonné ( $1^{\text {er }}$ niveau) fait apparaître. Le développement opérationnel (praxis) de cette approche méthodologique répond à la thèse centrale (theoria) du professeur Forrester selon laquelle la structure du système était responsable de sa dynamique et que, si cette dynamique avait des propriétés non désirées, il convenait alors d'intervenir en modifiant la structure ou les règles de comportement. On voit bien alors que, parti du principe théorique selon lequel on peut mettre en correspondance la structure du système et sa dynamique, on aboutit à l'idée, que l'on peut modifier la structure de sorte que le fonctionnement soit plus satisfaisant (décision de changement que nous avons nommée « ré-équilibrage »), ce qui fait évoluer le modèle et ainsi de suite.

Nous avons ainsi clairement illustré l'apport de la démarche appliquant la dynamique des systèmes à la gestion prévisionnelle des aléas dans la vie quotidienne des entreprises et des organisations. Les perspectives s'inscrivent directement dans la généralisation de l'appréhension de ces aléas à de plus subtiles perturbations peu prévisibles mais à très fort impact, ces changements inattendus de paradigme, d'usage, de produits (les « Cygnes noirs »). profonds impacts. Les détecter, mesurer leurs (C) 2018 ISTE OpenScience - Published by ISTE Ltd. London, UK - openscience.fr 
impacts et mener les actions nécessaires pour s'y préparer, s'y adapter par des transformations profondes, les pallier dans le court terme, doit être l'un des buts de la démarche de « management des futurs » dans les organisations économiques, entreprises, villes, régions, groupements de toutes natures.

\section{Bibliographie}

[ASH 56 ] ASHBY W.R., Introduction to Cybernetics, Chapman \& Hall, 1956

[BER 72] BERTALANFFY L. von, Théorie générale des systèmes, 1972

[CHA 84] CHAMUSSY H., GUERIN J-P., LE BERRE M., UVIETTA P, « La dynamique de systèmes : une méthode de modélisation des unités spatiales », Espace géographique, vol 13, n² 2, pp 81-93, 1984

[CHE 97] CHERY Jean-Pierre, « Pour un modèle spatial en zone frontalière franco-suisse : la frontière suisse considérée comme inertie spatiale et comme forme abstraite », DOI : 1.3406/globe.1997.1382

[COU 63] COUFFIGNAL L., La cybernétique, PUF, Collection Que Sais-je ?, nº 638, 1963

[DEN 16] DENEAULT D., Gouvernnance, in DENAULT D., La Médiocratie, Québec : Lux, 2016

[FIM 86] Guide méthodologique FIMTM, (FIM) et ADJ, 1986

[FOR 61] FORRESTER J.W., Industrial Dynamics, Waltham, MA: Pegasus Communications, 1961

[FOR 68] FORRESTER J.W., Principles of Systems, Cambridge : Productivity Press, 1968

[FOR 69] FORRESTER J.W., Urban Dynamics, Pegasus Communications, 1969

[FOR 73] FORRESTER J.W., World Dynamics, Pegasus Communications, 1973

[GAC 08] GACOGNE V., BOSSIN P., DHERVE S., «Pérennisation et villes durables. Le modèle DYNACITE. Simulation des jeux d'acteurs par une modélisation en dynamique des systèmes ", Rapport final pour l'Agence de l'Environnement et de la maîtrise de l'énergie, Programme de recherche PREDIT 3 2002-2007, 2008

[GAR 16] GARBOLINO E., CHERY J-P., GUAMIERI F., "A Simplified Approach to Risk Assessment Based on System Dynamics: An Industrial Case Study”, Risk Analysis, vol 36, n 1, January 2016

[JAN 03] JANCOVICI J.M., (2003), Qu’y a-t-il donc dans le "Rapport du Club de Rome”?, 2003, http://www.manicore.com/documentation/clubrome.html. Consulté le 19 mars 2009

[KAR 04] KARSKY M., DONNADIEU G., La systémique, penser et agir dans la complexité, Paris : Edition Liaisons, 2004

[MEA 72] MEADOWS D.H., MEADOWS D., RANDERS J., The Limits to Growth, New York Universe Books, 1972

[MEA 04] MEADOWS D.H., MEADOWS D., RANDERS J., The Limits to Growth: The 30-year Update, Chelsea Green Publishing, 2004, traduction en français par DELAUNAY J., Halte à la croissance

[MOI 84] LE MOIGNE J.L., Théorie du système général, Paris : PUF, 1984

[MOR77] MORIN E., La méthode, Seuil, Paris, 197 à 2004

[PAU 98] PAULRE Bernard. 1998. "La dynamique des systèmes, quarante ans après". In La dynamique des systèmes, THIEL D. (Dir.), Paris: Hermès, 1998. pp.16-24

[PRO 05] PROVITOLO Damienne, "Un exemple d'effets de domino: la panique dans les catastrophes urbaines », Cybergéo, European Journal of Geography, article 328, 29 novembre 2005

[ROS 75] ROSNAY J. de, Le macroscope, Le Seuil, Paris, 1975

[SAU 01] SAULAIS P., WENSINK E, "On the increasing importance of simulations in the conception of new naval radar systems", International Conference on Radar, Beijing, China, 2001

[SAU 13] SAULAIS P.. Application de la gestion des connaissances à la créativité des experts et à la planification de la $\mathrm{R}$ $\& \mathrm{~T}$ en milieu industriel de haute technologie, Thèse de doctorat, Télécom Ecole de Management, Evry (France).

[SEM 04] SEMAI L, «Développement durable et territoire», 2004, mis en ligne le 20 septembre 2007, URL: http://developpementdurable.revues.org/document4422.htm,.consulté le 19 mars 2009.

[STA 13] STAUNE J., Les clés du futur, Paris : Plon, 2013 
[STE 00] STERMAN John D., Business Dynamics - Systems Thinking and Modeling for a Complex World, USA: McGraw-Hill, 2000

[TAL 10] TALEB N., The Black Swan : The Impact of the highly improbable, 2008, Traduction française : Le cygne noir ou la puissance de l'imprévisible, Paris : Les Belles Lettres, 2010

[THI 98] THIEL D., La dynamique des systèmes, THIEL D., Paris: Hermès, 1998

[WIE 48] WIENER N. (1948), Cybernetics, or Control and Communication in the Animal and the Machine, The MIT Press (Cambridge, Mass.) et Wiley (New York), 1948

[WIE 52] WIENER N., Cybernétique et société, Union Générale d'Editions, Collection 10/18, 1952, Rééd. 1971Traduction française de The Human Use of Human Beings, Houghton Miftlin, Boston, 1950 